\title{
Perceptions of vascular access for intravenous systemic therapy and risk factors for lymphedema in early-stage breast cancer- a patient survey
}

\author{
N. LeVasseur $\mathrm{MD}^{*}$ C. Stober, ${ }^{+}$M. Ibrahim, ${ }^{*}$ S. Gertler $\mathrm{MD}^{*}{ }^{*}$ J. Hilton $\mathrm{MD}^{*+}{ }^{* \dagger}$ A. Robinson $\mathrm{MD}_{1}^{\neq}$ \\ S. McDiarmid RN, ${ }^{\S}$ D. Fergusson $\mathrm{PhD}^{+} \| \mathrm{S}$. Mazzarello, ${ }^{+}$B. Hutton $\mathrm{PhD}_{1}^{+} \|$A.A. Joy MD, \# \\ M. Mclnnes $\mathrm{MD}^{+* *}$ and M. Clemons $\mathrm{MD}^{*+\|}$
}

\begin{abstract}
Background The choice of vascular access for systemic therapy administration in breast cancer remains an area of clinical equipoise, and patient preference is not consistently acknowledged. Using a patient survey, we evaluated the patient experience with vascular access during treatment for early-stage breast cancer and explored perceived risk factors for lymphedema.

Methods Patients who had received systemic therapy for early-stage breast cancer were surveyed at 2 Canadian cancer centres.

Results Responses were received from 187 patients (94\%). The route of vascular access was peripheral intravenous line (IV) in $24 \%$, a peripherally inserted central catheter (PICC) in $42 \%$, and a surgically inserted central catheter (port) in $34 \%$. Anthracycline-based regimens were associated with a greater use of central vascular access devices (cvADsthat is, a PICC or port; 86/97, 89\%). Trastuzumab use was associated with greater use of ports (49/64, 77\%). Although few patients $(7 \%)$ reported being involved in the decisions about vascular access, most were satisfied or very satisfied (88\%) with their access type. Patient preference centred mainly on avoiding delays in the initiation of chemotherapy. Self-reported rates of complications (183 evaluable responses) were infiltration with peripheral IVs (9/44, 20\%), local skin infections with PICCs (7/77, 9\%), and thrombosis with ports (4/62,6\%). Perceived risk factors for lymphedema included use of the surgical arm for blood draws (117/156, 75\%) and blood pressure measurement (115/156, 74\%).
\end{abstract}

Conclusions Most patients reported being satisfied with the vascular access used for their treatment. Improved education and understanding about the evidence-based requirements for vascular access are needed. Perceived risk factors for lymphedema remain variable and are not evidence-based.

Key Words Early-stage breast cancer, patient surveys, vascular access

Curr Oncol. 2018 Aug;25(4):e305-e310

www.current-oncology.com

\section{BACKGROUND}

In patients with early-stage disease, adjuvant intravenous chemotherapy regimens have substantially improved breast cancer-specific survival ${ }^{1}$. Despite changes in recent years in the types of adjuvant chemotherapy used-such as the increased use of non-anthracycline-containing regimens (for example, docetaxel-cyclophosphamide)- and longer treatment durations (for example, 1 year of adjuvant trastuzumab for HER2-positive disease), the optimal route of venous access remains unknown ${ }^{2}$. Every vascular access choice, be it a peripheral intravenous (IV) line or a central vascular access device (CVAD) such as a peripherally inserted central catheter (PICC) or a surgically inserted central line (port), is associated with its own benefits and risks ${ }^{2}$. Peripheral IVs can be easily inserted at 
a low cost, but they can result in sclerosis of the veins and extravasation. A PICc can be used for weeks to months, but they are associated with physical limitations because of their external nature and with a greater risk of infectious and thrombotic complications. A port, which is completely implanted, can last for months to years, but involves a surgical procedure, greater cost, and an increased risk for thrombosis. Reliable vascular access is thought to improve the patient experience by reducing the number of peripheral IV insertion attempts and reducing the risk of peripheral phlebitis and chemotherapy extravasation ${ }^{3}$.

Given the perceived complexity and expertise required, the choice of vascular access route has typically been driven by the medical team in discussion with the patient. Although likely considered in the decision-making process, rates of potential complications and patient preferences might not be consistently considered in detailed discussions ${ }^{2,4}$. Given the variability in potential vascular access choices and patient preferences, we designed a survey to describe the patient's unique experience with vascular access during treatment for early-stage breast cancer, including their preferences, levels of satisfaction, perceived rates of complications, and risk factors for lymphedema. The findings are meant to improve vascular access strategies and the patient experience by allowing the health care team to identify evidence-based needs. The findings will also guide the development of educational materials addressing current knowledge gaps.

\section{METHODS}

\section{Questionnaire Design and Distribution}

The survey was developed by clinicians and researchers with expertise in survey design, medical oncology, epidemiology, and knowledge translation. Important themes were identified, and survey questions were drafted and reviewed by members of the research team. To add validity before implementation, the completed survey (supplementary Appendix 1) was then piloted with 3 patients. Recruitment for the survey ran for 14 weeks from April to June 2016. The survey and related documentation were reviewed and approved by the Ottawa Health Science Network Research Ethics Board. Verbal consent was obtained from all individual participants included in the study.

Patients with early breast cancer who had received or were currently receiving IV chemotherapy at 2 Canadian cancer centres (The Ottawa Hospital Cancer Centre and the Irving Greenberg Family Cancer Centre, Ottawa) were approached at the time of routine follow-up. A member of the health care team asked about their interest in participating in the survey. Interested individuals were given an information sheet about the study and a copy of the survey for completion. Patients were asked for their age, type of chemotherapy received, type of vascular access used, preferences for vascular access, and details about who made the final access decision. Patients were also surveyed about complications encountered with vascular access and the perceived risk factors for lymphedema. No signed consent for the study was sought; consent was considered to have been provided if a patient completed and submitted the survey. Of 200 surveys prepared for distribution, 187 were completed and received by the research team.

\section{Statistical Analysis}

The survey consisted of close-ended multiple-choice questions with the option to select as many answers as appropriate, and hybrid questions asking the respondent to rate items in order of importance or to estimate rates. Responses were summarized descriptively as proportions with their 95\% confidence intervals in Microsoft Excel 2010 (Microsoft Corporation, Redmond, WA, U.S.A.). Free-text responses were collated to directly address patient concerns or comments for future use in a quality improvement project. No formal hypotheses were tested.

\section{RESULTS}

\section{Demographics}

The survey response rate was $94 \%$ (187/200). All respondents were attending either The Ottawa Hospital Cancer Centre or the Irving Greenberg Family Cancer Centre. Mean age in the respondent group was 55 years (range: 23-90 years).

\section{Chemotherapy Received}

When asked which chemotherapy regimen they had received, 151 of 187 patients $(81 \%)$ recalled this information and provided responses (Table I). The most common regimens were FEC-D [fluorouracil-epirubicin-cyclophosphamide followed by docetaxel (53/151,35\%)], DC [docetaxel-cyclophosphamide (42/151, 28\%)], AC-T [doxorubicin-cyclophosphamidepaclitaxel $(37 / 151,25 \%)]$ and AC-D [doxorubicincyclophosphamide-docetaxel $(8 / 151,5 \%)]$. The remaining $11 \%(16 / 151)$ reported receiving another regimen that was not among the options provided. Although patients were asked to select only 1 answer, 5 respondents selected 2 answers. Patients were also asked about trastuzumab, resulting in 173 responses, with 64 (37\%) reporting that they received trastuzumab; $82(47 \%)$, that they did not receive trastuzumab; and $28(16 \%)$, that they were unsure. Although patients were asked to select only 1 answer, 1 respondent selected both "no" and "unsure." The remaining patients did not respond (14/187, 7\%).

\section{Decisions About Vascular Access}

Of the 187 completed surveys, 179 contained responses about the decision for vascular access. Of those 179 respondents, 74\% (133/179) recalled a discussion about vascular access. The remaining respondents either believed that there had been no discussion of vascular access (36/179, $20 \%)$ or were unsure $(10 / 179,6 \%)$. When asked who made the decision for vascular access, patients indicated that the decision was based primarily on a recommendation from the medical oncologist $(118 / 153,77 \%)$, on their personal preference $(11 / 153,7 \%)$, or on a recommendation from the clinic nurse $(6 / 153,4 \%)$ or a chemotherapy nurse $(8 / 153$, $5 \%$ ). A small proportion reported that they did not have a central line or that the decision was made by another member of the team $(10 / 153,7 \%)$.

When asked how a range of factors would affect their choice between a PICC or a port, the only factor with a large 
TABLE I Characteristics of 187 survey responders

\begin{tabular}{|c|c|c|c|c|c|}
\hline \multirow[t]{2}{*}{ Characteristic } & \multirow{2}{*}{$\begin{array}{c}\text { Responses } \\
\text { (n) }\end{array}$} & \multicolumn{4}{|c|}{ Response by access type } \\
\hline & & Overall & PIV & PICC & PORT \\
\hline \multicolumn{6}{|l|}{ Age (years) } \\
\hline Mean & & 55 & & & \\
\hline Range & & $23-90$ & & & \\
\hline Chemotherapy regimen $[n(\%)]$ & $151^{\mathrm{a}}$ & & & & \\
\hline DC & & $42(28)$ & $22 / 36(61)$ & $4 / 36(11)$ & $10 / 36(28)$ \\
\hline FEC-D & & $53(35)$ & 6/53 (11) & $28 / 53(53)$ & 19/53 (36) \\
\hline AC-T & & $37(25)$ & $3 / 36(8)$ & $23 / 36(64)$ & $10 / 36(28)$ \\
\hline AC-D & & $8(5)$ & $2 / 8(25)$ & $3 / 8(38)$ & $3 / 8(38)$ \\
\hline Other & & $16(11)$ & & & \\
\hline Trastuzumab [n(\%)] & $173^{b}$ & & & & \\
\hline Yes & & $64(37)$ & 8/64 (13) & $7 / 64(11)$ & $49 / 64(77)$ \\
\hline No & & $82(47)$ & $27 / 82(33)$ & $50 / 82(61)$ & $6 / 82(7)$ \\
\hline Unsure & & $28(16)$ & & & \\
\hline Vascular access $[n(\%)]$ & 183 & & & & \\
\hline PIV & & $44(24)$ & & & \\
\hline PICC & & $77(42)$ & & & \\
\hline PORT & & $62(34)$ & & & \\
\hline
\end{tabular}

a Five patients selected more than 1 answer.

b One patient selected 2 answers.

PIV = peripherally inserted intravenous line; PICC = peripherally inserted central catheter; PORT = surgically implanted central catheter; DC = docetaxel-cyclophosphamide; FEC-D = 5-fluorouracil-epirubicin-cyclophosphamide, then docetaxel; AC-T = doxorubicin-cyclophosphamide, then paclitaxel; AC-D = doxorubicin-cyclophosphamide, then docetaxel.

impact on the choice of access method by patients was the possibility of a delay in starting chemotherapy $(76 / 158$, $48 \%$ ). Other factors such as the risk of infection or of thromboembolism most often had just a moderate impact at $41 \%$ $(65 / 158)$ and $43 \%(66 / 155)$ respectively (Figure 1$)$. Factors reported as having no impact on the decision about vascular access (Figure 1) included difficult removal (68/157, $43 \%)$, need for frequent flushing (96/154, 62\%), physical appearance $(106 / 156,68 \%)$, inconvenience for showering or swimming $(76 / 157,48 \%)$, and possibility of the device being accidentally pulled out (111/153, 73\%).

With respect to access type received, 183 responses were obtained (Table I). Of those 183 patients, $24 \%(n=44)$ reported receiving all chemotherapy through a peripheral IV that required an average of 2 attempts at insertion at each chemotherapy visit. Most patients received chemotherapy through a CVAD: $42 \%(n=77)$ through a PICC and $34 \%$ $(n=62)$ through a port.

\section{Choice of Vascular Access Based on Chemotherapy Regimen}

The choice of vascular access was also evaluated based on chemotherapy regimen received (Table I). Enough information for this analysis was provided on 133 surveys. Patients were more likely to receive Dc chemotherapy by peripheral IV $(22 / 36,61 \%)$ than by PICC $(4 / 36,11 \%)$ or port $(10 / 36,28 \%)$. Anthracycline-taxane combination regimens were more likely to be delivered by PICC $(54 / 97,56 \%)$ or port $(32 / 97,33 \%)$ than by peripheral IV $(11 / 97,11 \%)$.
For the use of trastuzumab, 147 evaluable responses were available (Table I). A higher proportion of patients received trastuzumab-containing chemotherapy by port $(49 / 64,77 \%)$ than by either PICC $(7 / 64,11 \%)$ or peripheral IV $(8 / 64,13 \%)$. When trastuzumab was not part of the regimen, fewer ports were used $(6 / 82,7 \%)$; PICCs $(50 / 82$, $61 \%)$ and peripheral IVs $(27 / 82,33 \%)$ were more frequent.

When comparing how the use of trastuzumab with either a non-anthracycline-containing or an anthracyclinecontaining chemotherapy regimen affected the choice of vascular access, the addition of trastuzumab to a nonanthracycline regimen (for example, DC) changed the choice of vascular access from predominantly peripheral IV $(14 / 16,88 \%)$ to a port $(9 / 13,69 \%)$. Similarly, for regimens containing both an anthracycline and a taxane, the addition of trastuzumab changed the most common type of access from a PICC $(41 / 54,76 \%)$ to a port $(23 / 29,79 \%)$.

\section{Rates of Complications}

Overall, $74 \%$ of respondents to the questions about complications (136/183) reported no complications with their vascular access during treatment (Table II). With respect to delays, patients reported similar rates in the range of $3 \%-5 \%$ for all vascular access types (peripheral IV: $2 / 44$, 5\%; PICC: $4 / 77,5 \%$; port: $2 / 62,3 \%$ ). Of patients receiving treatment through a peripheral IV, 20\% (9/44) reported chemotherapy leaking under the skin, and $9 \%$ reported (4/44) self-perceived poor IV access. The rate of local skin infection varied from $9 \%(7 / 77)$ with a PICc to $5 \%(3 / 62)$ with 


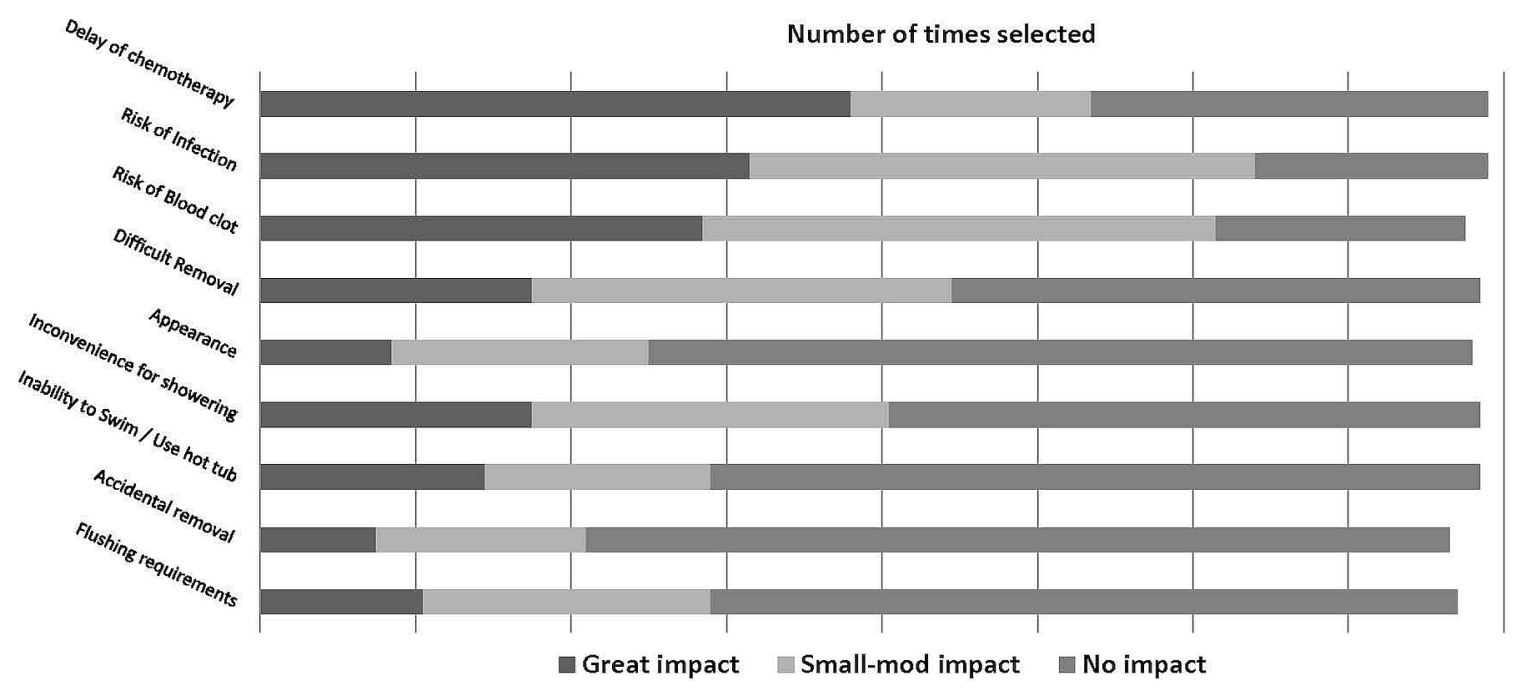

FIGURE 1 Factors influencing patient choice for intravenous access. Mod = moderate.

TABLE II Complication rates self-reported by 183 patients

\begin{tabular}{|c|c|c|c|c|c|c|}
\hline \multirow[t]{3}{*}{ Variable } & \multicolumn{6}{|c|}{ Patient group } \\
\hline & \multicolumn{2}{|c|}{ PIV } & \multicolumn{2}{|c|}{ PICC } & \multicolumn{2}{|c|}{ PORT } \\
\hline & $(n)$ & $(\%)$ & $(n)$ & $(\%)$ & $(n)$ & $(\%)$ \\
\hline Respondents & 44 & 24 & 77 & 42 & 62 & 34 \\
\hline Delay in chemotherapy start waiting for line insertion & 2 & 5 & 4 & 5 & 2 & 3 \\
\hline Chemotherapy leaking under the skin & 9 & 20 & 0 & 0 & 0 & 0 \\
\hline Intravenous line not working & 4 & 9 & 0 & 0 & 0 & 0 \\
\hline Infection in the skin & 0 & 0 & 7 & 9 & 3 & 5 \\
\hline Blood clot requiring anticoagulation & 0 & 0 & 3 & 4 & 4 & 6 \\
\hline Line pulled out accidentally & 1 & 2 & 7 & 9 & 0 & 0 \\
\hline Difficult removal of the line after chemotherapy completion & 0 & 0 & 4 & 5 & 2 & 3 \\
\hline
\end{tabular}

PIV = peripherally inserted intravenous line; PICC = peripherally inserted central catheter; PORT = surgically implanted central catheter.

a port, with no infections being reported for peripheral IV $(0 / 44)$. The rate of venous thromboembolism was highest, at $6 \%(4 / 62)$, in patients with a port; the rate in the PICC group was $4 \%$ (3/77). Mechanical factors, such as the access device accidentally being pulled out, was reported more often with PICCs $(7 / 77,9 \%)$ than with peripheral IVs $(1 / 44$, $2 \%)$. Difficult device removal was reported in $5 \%$ of patients with a PICC (4/77) and in $3 \%$ of patients with a port $(2 / 62)$.

\section{Patient Satisfaction and Comments}

When asked to rate their satisfaction with the vascular access used during their treatment, most patients reported being very satisfied $(109 / 179,61 \%)$ or satisfied $(49 / 179,27 \%)$; only a few reported being somewhat satisfied (16/179, 9\%) or not satisfied (5/179, 3\%). When given the opportunity to advise future patients starting chemotherapy about access type, most respondents recommended the type of vascular access that they had received and recommended that patients follow the advice of their oncology team. Those responses were consistent whether patients had a peripheral IV or a CVAD. Examples of patient comments included these:

I would suggest to have a PICC line. I don't like needles, and [I] find this less stressful and more convenient. Caring for the line is not difficult.

Choose what works best for you. Listen to your doctor. Sometimes simple is best-i.e., IV was less stressful and less traumatic for me.

Ask lots of questions about the PICc line so there are no surprises. It was a surprise to me that I needed one, which was upsetting at the time. It did ultimately serve a good purpose and was easier to manage with showering/clothing than anticipated.

Patient-Perceived Risk Factors for Lymphedema

Most patients recognized that lymphedema was a risk during treatment for their breast cancer (168/177, 95\%). 
Patients were also asked to list any member of their care team (multiple responses permitted) who educated them about the risk for lymphedema. Surgeons were listed most often $(90 / 169,53 \%)$, followed by the surgical nurse $(77 / 169$, $46 \%)$, chemotherapy nurses $(74 / 169,44 \%)$, and medical oncologists $(72 / 169,43 \%)$. Other members of the care team-for example, physiotherapists $(38 / 169,22 \%)$ and radiation oncologists $(32 / 169,19 \%)$-were less often listed as providing this education. Patients also reported being told about lymphedema by other cancer patients $(26 / 169,15 \%)$, the Internet $(37 / 169,22 \%)$, or other sources $(2 / 169,1 \%)$.

Patients who recalled being told to avoid blood draws or blood pressure readings using their surgical arm were asked if they knew the reason for that advice. Most recalled that the reason was to avoid the development of lymphedema $(144 / 178,81 \%)$. A small number reported being unsure $(17 / 178,10 \%)$ or never having been told to avoid blood draws or blood pressure readings (15/178, 8\%). A few respondents believed that such use of their surgical arm might increase the chance of the cancer returning $(2 / 178,1 \%)$.

When asked about specific factors that would increase the chance of lymphedema developing (multiple responses accepted), the most frequently identified risk factors included blood draws (117/156, 75\%), blood pressure measurements $(115 / 156,74 \%)$, and axillary lymph node dissection $(80 / 156,51 \%)$. Other factors such as lifting heavy objects $(67 / 156,43 \%)$, infection in the arm $(62 / 156,40 \%)$, chemotherapy administration in the surgical arm (52/156, $33 \%)$, and use of hot tubs or saunas $(51 / 156,33 \%)$ were also selected. The factor least often selected was radiation to the axilla (41/156, 26\%).

\section{DISCUSSION}

Results from the present study highlight the fact that significant clinical equipoise still exists with respect to the use of vascular access devices in patients receiving chemotherapy for early-stage breast cancer. Heterogeneity in access is seen for the various chemotherapy regimens, but also for the same regimens. Use of either an anthracycline or trastuzumab was associated with a higher proportion of CVAD use; in the case of trastuzumab, the cVAD was more commonly a port. Patients rarely reported being involved in the decision about the type of vascular access; nevertheless, their satisfaction with their access remained high. Indeed, the only factor that stood out as affecting the patient's decision for vascular access was avoidance of delay in starting chemotherapy.

With respect to complication rates, the risk of chemotherapy infiltration with a peripheral IV was self-reported as $20 \%$. The rates of extravasation reported in the literature range from $1 \%$ up to $7 \%^{5}$. The high rate reported here might reflect recall bias or a difference in the perception of the definition of extravasation. With respect to skin infection, rates of $9 \%$ and $5 \%$ were reported for PICCs and ports respectively. Although no direct comparison can be made with patient-reported rates of complications, it is important to note that one systematic review comparing the risks of infection with PICcs and with other central vascular catheters reported central line-associated bloodstream infections in $5.2 \%$ of inpatients and $0.5 \%$ of outpatients ${ }^{6}$.
Similarly, in our survey, self-reported rates of thrombosis were $4 \%$ for patients with a PICC and $6 \%$ for those with a port, which are again higher than the rates reported in systematic reviews ${ }^{7,8}$.

Finally, with respect to lymphedema, most patients recognized the risk. They most often reported being informed by their surgeon and surgical nurse, closely followed by their chemotherapy nurse and their medical oncologist. Current evidence and best practice for the prevention of lymphedema are based on identifying the actual risk. To date, the identified risk factors include axillary surgery, axillary radiotherapy, obesity, and prior cellulitis. The perception that blood draws and blood pressure measurements using the surgical arm increase the risk for lymphedema continue to be reported by patients, despite published data that suggest otherwise ${ }^{9-14}$. Other historical risk factors are also frequently reported by patients ${ }^{15}$. Recognized risk factors such as radiation to the axilla were less often reported by patients ${ }^{16}$.

Many myths about the risk factors for the development of lymphedema continue to be perpetuated. Based on the results of the present survey, educational materials about the risks for lymphedema seem to be lacking, and efforts should be made to provide patients with reliable information. In addition, any strategy for dispelling the myths will require the involvement of multiple stakeholders.

When looking at the choice of chemotherapy regimen, it appears that our centres used FEC-D more often in the adjuvant setting than has been reported in systematic reviews ${ }^{17}$. The reported rates of trastuzumab use were also slightly higher than published rates, although many patients were unsure and likely did not receive trastuzumab ${ }^{18}$. Also, women with HER2-positive disease might be more likely to receive chemotherapy and might therefore have been overrepresented in this sample of patients ${ }^{19}$.

\section{Study Limitations}

There are clearly limitations to any survey. This relatively small patient sample was accrued from 2 cancer centres in a single Canadian city, albeit with a good response rate. There also appears to be an overrepresentation of HER2-positive patients. Because the survey was anonymous, the information provided could not be verified using patient charts. As a result, responses about complication rates should be interpreted with caution. Well-designed randomized controlled trials could ultimately help answer these questions with greater certainty. Our group is currently performing two such studies (see NCT02632435, NCT02688998, and NCT02632435 at http://ClinicalTrials.gov).

\section{CONCLUSIONS}

Vascular access strategies are an integral component of the patient experience and treatment outcomes. Our study supports the fact that anthracycline or trastuzumab chemotherapy is associated with greater use of cvaDs. It also draws attention to the fact that incorrect perceptions about risk factors for lymphedema continue to be propagated and that reliable patient education materials are necessary. Ultimately, the results of the present study provided the rationale for two ongoing clinical trials that, hopefully, 
will answer many important questions related to vascular access for breast cancer patients.

\section{ACKNOWLEDGMENTS}

We thank our patients and their families for their assistance with this project. We also thank the physicians who allowed us to approach their patients (Drs. Roanne Segal and Susan Dent) and Miguel de Costa e Silva for assistance with data collection. Funding for this project was provided through core institutional funds for The Rethinking Clinical Trials Program based at The Ottawa Hospital Research Institute.

\section{CONFLICT OF INTEREST DISCLOSURES}

We have read and understood Current Oncology's policy on disclosing conflicts of interest, and we declare the following interests: SM holds an advisory role with AngioDynamics. All other authors declare that they have no conflicts to disclose.

\section{AUTHOR AFFILIATIONS}

*Division of Medical Oncology and Department of Medicine, The Ottawa Hospital and University of Ottawa, Ottawa; ${ }^{\dagger}$ The Ottawa Hospital Research Institute, Ottawa; ${ }^{\ddagger}$ Division of Medical Oncology, Cancer Centre of Southeastern Ontario, Kingston; ${ }^{\S}$ Department of Nursing, The Ottawa Hospital, Ottawa; and "Clinical Epidemiology Program, The Ottawa Hospital Research Institute, Ottawa, ON; \# Department of Oncology, Division of Medical Oncology, University of Alberta, Cross Cancer Institute, Edmonton, $\mathrm{AB}$; **Department of Radiology, University of Ottawa, Ottawa, ON.

\section{REFERENCES}

1. Early Breast Cancer Trialists' Collaborative Group. Comparisons between different polychemotherapy regimens for early breast cancer: meta-analyses of long-term outcome among 100,000 women in 123 randomised trials. Lancet 2012;379:432-44.

2. AwA, Carrier M, Koczerginski J, McDiarmid S, Tay J. Incidence and predictive factors of symptomatic thrombosis related to peripherally inserted central catheters in chemotherapy patients. Thromb Res 2012;130:323-6.

3. Goolsby TV, Lombardo FA. Extravasation of chemotherapeutic agents: prevention and treatment. Semin Oncol 2006;33:139-43.

4. Scott WL. Central venous catheters. An overview of Food and Drug Administration activities. Surg Oncol Clin N Am 1995;4:377-93.

5. Pérez Fidalgo JA, García Fabregat L, Cervantes A, Margulies A, Vidall C, Roila F on behalf of the Esmo Guidelines Working Group. Management of chemotherapy extravasation: ESMO-EONS clinical practice guidelines. Eur J Oncol Nurs 2012;16:528-34.

6. Chopra V, O'Horo JC, Rogers MA, Maki DG, Safdar N. The risk of bloodstream infection associated with peripherally inserted central catheters compared with central venous catheters in adults: a systematic review and meta-analysis. Infect Control Hosp Epidemiol 2013;34:908-18.

7. Chopra V, Anand S, Hickner A, et al. Risk of venous thromboembolism associated with peripherally inserted central catheters: a systematic review and meta-analysis. Lancet 2013;382:311-25.

8. Johansson E, Hammarskjold F, Lundberg D, Arnlind MH. Advantages and disadvantages of peripherally inserted central venous catheters (PICC) compared to other central venous lines: a systematic review of the literature. Acta Oncol 2013;52:886-92.

9. Ferguson CM, Swaroop MN, Horick N, et al. Impact of ipsilateral blood draws, injections, blood pressure measurements, and air travel on the risk of lymphedema for patients treated for breast cancer. J Clin Oncol 2016;34:691-8.

10. United States, Department of Health and Human Services, National Institutes of Health, National Cancer Institute (NCI). Lymphedema (PDQ)-Health Professional Version [Web page]. Bethesda, MD: NCI; 2015. [Available at: https://www.cancer. gov/about-cancer/treatment/side-effects/lymphedema/ lymphedema-hp-pdq; cited 26 September 2017]

11. Meneses KD, McNees MP. Upper extremity lymphedema after treatment for breast cancer: a review of the literature. Ostomy Wound Manage 2007;53:16-29.

12. Norman SA, Localio AR, Potashnik SL, et al. Lymphedema in breast cancer survivors: incidence, degree, time course, treatment, and symptoms. J Clin Oncol 2009;27:390-7.

13. Fu MR, Deng J, Armer JM. Putting evidence into practice: cancer-related lymphedema. Clin J Oncol Nurs 2014;18 (suppl):68-79.

14. Oncology Nursing Society (ons). Lymphedema [Web page]. Pittsburgh, PA: ons; 2017. [Available at: https://www.ons.org/ practice-resources/pep/lymphedema; cited 27 June 2017]

15. Bicego D, Brown K, Ruddick M, Storey D, Wong C, Harris SR. Exercise for women with or at risk for breast cancer-related lymphedema. Phys Ther 2006;86:1398-405.

16. Lucci A, McCall LM, Beitsch PD, et al. on behalf of the American College of Surgeons Oncology Group. Surgical complications associated with sentinel lymph node dissection (SLND) plus axillary lymph node dissection compared with SLND alone in the American College of Surgeons Oncology Group Trial Z0011. J Clin Oncol 2007;25:3657-63.

17. Fujii T, Le Du F, Xiao L, et al. Effectiveness of an adjuvant chemotherapy regimen for early-stage breast cancer: a systematic review and network meta-analysis. JAMA Oncol 2015;1:1311-18.

18. Moasser MM, Krop IE. The evolving landscape of HER2 targeting in breast cancer. JAMA Oncol 2015;1:1154-61.

19. Broglio KR, Quintana M, Foster M, et al. Association of pathologic complete response to neoadjuvant therapy in HER2-positive breast cancer with long-term outcomes: a meta-analysis. JAMA Oncol 2016;2:751-60. 